\title{
CATEGORIZACIÓN DE RECEPTORES $\alpha 1$-ADRENÉRGICOS EN EL DETRUSOR DE PACIENTES CON HBP OBSTRUCTIVA. ESTUDIO INICIAL EN MODELO ANIMAL DE EXPERIMENTACIÓN
}

\author{
C. GONZÁleZ ENGUITA, R. VELA NAVARRETE, A. SALCEDO DE DIEGO, \\ F. PÉREZ MARTÍNEZ, M. RAMÍREZ PÉREZ DEL YERRO, M.J. CANCHO GIL, \\ F. CRESPÍ MARTÍNEZ, I. BRAVO FERNÁNDEZ, J. CABRERA PÉREZ
}

Cátedra y Servicio de Urología. Fundación "Jiménez Díaz". Universidad Autónoma. Madrid.

Actas Urol Esp. 27 (9): 684-691, 2003

\section{RESUMEN}

CATEGORIZACIÓN DE RECEPTORES $\alpha 1$-ADRENEERGICOS EN EL DETRUSOR DE PACIENTES CON HBP OBSTRUCTIVA. ESTUDIO INICIAL EN MODELO ANIMAL DE EXPERIMENTACIÓN

INTRODUCCIÓN: Los antagonistas de los receptores adrenérgicos $\alpha 1$ han demostrado su acción en pacientes con obstrucción al flujo vesical (Hiperplasia Benigna de Próstata-HBP) al mejorar los sintomas obstructivos e irritativos. Si bien es conocido que los receptores $\alpha$ la son los responsables de la relajación del músculo liso de la próstata, es desconocido el mecanismo por el cual se mejoran los sintomas irritativos de responsabilidad vesical. Diferentes subtipos de receptores $\alpha 1$ podrian estar implicados. El objetivo de esta investigación es indagar en los cambios poblacionales de receptores $\alpha$-adrenérgicos en el detrusor obstruido, y determinar que subtipo es el que proporcionalmente aumenta en esta situación (obstrucción vesical al flujo miccional).

MATERIAL Y MÉTODOS: El estudio se realiza in vivo en un modelo animal de experimentación: conejos machos NZ (Nueva Zelanda). Un grupo de ellos es obstruido quirúrgicamente, a nivel del cuello vesical, mediante la técnica propuesta por Levin et al. ${ }^{1.2}$. Previa cateterización vesical con sonda $8 \mathrm{Fr}$, y a través de una incisión media abdominal baja se expone el cuello vesical, que será ligado con una seda de $2 / 0$, por debajo de los orificios ureterales, retirando la sonda al final de esta intervención. Después de 4 semanas se sacrifica el conejo con pentotal intracardiaco y se realiza cistectomía. El grupo control son otros conejos no sometidos a obstrucción vesical que se sacrifican también en este momento para estudio comparativo. Parte del detrusor es sometido a estudio anatomo-patológico y el resto a un estudio fisio-farmacológico en baños de órgano mediante agonistas (phenilefrina y noradrenalina) y antagonistas de adrenoceptores: WB4101, AH11101A y BMY7378, antagonistas respectivos de los receptores $\alpha$ la,b,d.

RESULTADOS: Los hallazgos anatomo-patológicos demuestran mayor grosor de la pared de la vejiga en los casos de obstrucción vesical. Los estudios fisio-farmacológicos ponen de manifiesto que la respuesta del detrusor al agonista selectivo $\alpha 1$-adrenérgico es mayor en los conejos sometidos a obstrucción vesical, sin embargo la contractilidad del detrusor está disminuida (KPSS). En condiciones de obstrucción son los receptores $\alpha$ ld los que se ven aumentados.

DISCUSIÓN Y CONCLUSIONES: Los estudios que pretenden diferenciar las distintas poblaciones de $\alpha$-adrenoceptores son estudios de binding (Malloy et al. $)^{3}$ que localizan y cuantifican los distintos subtipos de receptores presentes en el tejido sin tener en cuenta su actividad. Los ensayos isométricos y fisiofarmacológicos evalúan receptores activos, es decir, que responden a estimulos agonistas y antagonistas, lo que permite valorar con exactitud la actividad del detrusor. Los resultados obtenidos en este estudio de investigación, apoyan la hipótesis del incremento de receptores adrenérgicos $\alpha 1$ en el detrusor obstruido con una p altamente significativa, y coincidiendo con trabajos moleculares previos ${ }^{4}$, durante la obstrucción prostática la subpoblación predominante en la vejiga es la $\alpha$ ld. Estos hallazgos pueden tener implicaciones fisiopatológicas, clínicas y farmacológicas. Si esta hipótesis, demostrada en experimentación animal, se consigue demostrar en la fase humana de la investigación, el desarrollo farmacológico deberá localizarse no sólo en fármacos antagonistas selectivos de receptores $\alpha$ la (próstata), sino también de los $\alpha$ ld, con responsabilidad clínica vesical en situación de obstrucción vesical al flujo miccional (HBP y prostatismo).

PALABRAS CLAVE: Receptores $\alpha$-adrenérgicos. HBP (Hiperplasia Benigna de Próstata). Obstrucción vesical. Estudios fisio-farmacológicos "in vitro" e "in vivo". Fármacos $\alpha$-agonistas.

\section{ABSTRACT}

a1-ADRENERGIC RECEPTORS CATEGORISATION IN THE DETRUSOR OF PATIENTS WITH OBSTRUCTIVE BPH. EARLY STUDY IN EXPERIMENTAL MODELS

INTRODUCTION: The action of $\alpha 1$-adrenergic receptor antagonists in ameliorating irritation and obstruction in patients with bladder outlet obstruction (due to Benign Prostatic Hyperplasia-BPH) has been demonstrated. Although it is well known that $\alpha 1$-a receptors are responsible for prostate smooth muscle relaxation, the mechanism by which irritative bladder symptoms are improved is unknown. Different $\alpha 1$ receptor subtypes may be involved. The objective of this study is to investigate the changes in the $\alpha-$ adrenergic receptor populations in the obstructed detrusor, and to determine which subtype is proportionally increased in this situation (bladder outlet obstruction).

MATERIAL AND METHODS: This was an in vivo study in an experimental model: male NZ (New Zealand) rabbits. The bladder neck of one group of rabbits was obstructed surgically using the technique proposed by Levin et a $\mathrm{al}^{1,2}$. Subsequent to bladder catherisation with a $8 \mathrm{Fr}$ catheter, the bladder neck was exposed by means of a low medial abdominal incision. The bladder neck was tied with a $2 / 0$ silk thread below the ureteral orifices. The catheter was taken out at the end of the intervention. The rabbits were sacrificed after 4 weeks using intracardiac pentothal and cystectomy was performed. A control group that did not undergo bladder obstruction were also sacrificed at this time, for the comparative study. Part of the detrusor was used for a pathological study and the rest for a physio-pharmacological study in which the organ was placed in a bath of adrenoceptor agonists (phenylephrine and noradrenaline) and antagonists WB101, AH11101A and BMY7378 (antagonists of the $\alpha 1$ a, b, d, respectively).

RESULTS: The findings of the pathological study show that the bladder wall was thicker in the rabbits that underwent bladder obstruction. The physio-pharmacological studies demonstrate that the detrusor response to the selective $\alpha-1$ adrenergic agonist was greater in the rabbits that underwent bladder obstruction, however detrusor contractility was decreased (KPSS). With bladder obstruction the $\alpha 1 \mathrm{~d}$ receptors were increased.

DISCUSSION AND CONCLUSIONS: Receptor-binding studies (Malloy et al) ${ }^{3}$ aim to differentiate the $\alpha$-adrenoreceptor populations. These studies identify and quantitate the different receptor subtypes in tissue without taking into account their activity. The isometric and physio-pharmacological tests evaluate active receptors, i.e. those that respond to agonist and antagonist stimuli. This enables detrusor activity to be evaluated accurately. The results obtained in this investigational study support the hypothesis that there is a high statistically significant increase in the $\alpha 1$ adrenergic receptors in the obstructed detrusor. Furthermore, in agreement with previous molecular studies ${ }^{4}$, during prostate obstruction $\alpha$ ld is the predominate sub-population in the bladder. These findings may have patho-physiological, clinical and pharmacological implications. If this hypothesis which has been demonstrated in an experimental model, is also demonstrated in studies in humans, pharmacological development should not only be focussed on selective $\alpha$ la receptor antagonists (prostate) but also on those of $\alpha$ ld, for relieving symptoms in patients with bladder outlet obstruction (BPH and prostatism).

** Trabajo realizado con una ayuda de la FIU (Fundación para la Investigación en Urología).

Fase inicial: Experimentación Animal. 
L os sintomatología clínica que se asocia a la Hiperplasia Benigna de Próstata (HBP), resultante de la obstrucción vesical al flujo miccional, es una situación patológica muy común entre los varones. Los síntomas se diferencian y se agrupan en dos grandes categorias: aquellos de carácter obstructivo y los irritativos. Los primeros (dificultad al comienzo de la micción, chorro débil, micción prolongada, sensación de vaciado vesical incompleto tras la micción,...), están muy relacionados con la existencia del adenoma de próstata en el canal uretral, su compromiso ocupacional y del componente funcional y dinámico de todo el área cervico-uretral. La síntomatología irritativa (urgencia miccional, polaquiuria, nocturia,...), son la expresión clínica de los cambios originados por la obstrucción (HBP) en la dinámica vesical. Los pacientes con HBP y obstrucción vesical al flujo, pueden tener hiperactividad vesical que les provoca contracciones vesicales no controladas. En ocasiones la sintomatología irritativa puede persistir después de haberse resuelto la obstrucción con normalización de los valores de flujo urinario ${ }^{5}$.

Los avances más recientes en el conocimiento neurohumoral y la clínica diaria, han permitido establecer un concepto dinámico del prostátismo. La obstrucción prostática en HBP, no está motivada exclusivamente por la presencia física del adenoma de próstata en la zona uretral, sino también por el tono de todos los elementos incluidos en este área, especialmente del músculo liso del adenoma.

Desde la década de los setenta (s.XX), y gracias a la Investigación Farmacológica, se conoce la existencia de receptores y neurotransmisores autonómicos en el área cérvico-uretral y prostática. Es clínicamente conocido que el bloqueo del simpático a nivel de los receptores $\alpha 1$ mediante fármacos $\alpha 1$ antagonistas en esta zona, mejora considerablemente la sintomatología del paciente que sufre obstrucción prostática. Por estudios moleculares y de contractilidad, se ha demostrado que son los receptores $\alpha$ la los subtipos responsables de la contracción del músculo liso prostático $^{6}$.

Esta respuesta observada en el tratamiento del prostatismo, puede ser debida a la acción relajante de la obstrucción dinámica de la prós- tata, sin embargo el flujo miccional no mejora de manera tan activa. Mediante estudios "in vivo" en animales de experimentación (perros) y en el hombre (paciente prostático) se sabe que, la obstrucción vesical crónica provoca hipertrofia del detrusor ${ }^{7}$. Si bien los fármacos antagonistas selectivos de los $\alpha$ la mejoran la obstrucción, no parece que sean ellos los que actúen sobre los sintomas irritativos, por lo que se presume la responsabilidad de otros subtipos de receptores $\alpha 1$ y un cambio en la cuantía de los distintos receptores $\alpha 1$ en el detrusor en condiciones de obstrucción ${ }^{8}$.

Gracias a todos estos conocimientos y a la observación clínica alcanzada en el tratamiento de pacientes prostáticos con fármacos $\alpha$-antagonistas, se ha podido diseñar un Proyecto de Investigación Básica que lleva por título: "Categorización de Receptores $\alpha 1$ - adrenérgicos en el detrusor de pacientes con HBP obstructiva". En esta publicación se presenta la primera fase del proyecto, ya concluida, en un modelo animal de experimentación, y los objetivos iniciales conseguidos.

\section{MATERIAL Y MÉTODOS}

El título del proyecto de Investigación es: "Categorización de Receptores $\alpha 1$-adrenérgicos en el detrusor de pacientes con HBP obstructiva".

La hipótesis de trabajo es: "Indagar sobre la cuantía y signos de las poblaciones correspondientes a los receptores $\alpha$-adrenérgicos a nivel del detrusor".

El proyecto está planteado en dos etapas, inicialmente en animal de experimentación (conejos) y con posterioridad en pacientes varones.

\section{Grupo A:}

Animal de experimentación: Conejos machos de Nueva Zelanda (Conejos NZ). Peso: 3,5 Kg.

$\mathrm{El}$ conejo $\mathrm{NZ}$ es un animal económico y de fácil manejo quirúrgico.

Este grupo a su vez se subdivide en otros dos grupos:

1. Conejos con uropatía obstructiva provocada quirúrgicamente.

2. Conejos sin uropatía obstructiva (grupo Control). 


\section{Grupo B:}

Pacientes varones de igual edad y sin patología secundaria.

Éstos a su vez también se subdividen en otros dos grupos:

1. Pacientes con uropatía obstructiva e hipertrofia del detrusor.

2. Pacientes sin uropatía obstructiva (grupo control).

La primera fase del proyecto de investigación se desarrolla "in vivo" en modelo animal de experimentación (conejo NZ).

Estos conejos son sometidos a cirugía bajo anestesia general en el quirófano de Cirugía Experimental. Se les realiza una obstrucción mecánica del flujo miccional a nivel del cuello de la vejiga según la técnica de Robert Levin et al. ${ }^{1,2}$. Previa colocación de una sonda vesical de $8 \mathrm{~F}$ de calibre, se realiza una incisión media abdominal baja para exponer la zona del cuello de la vejiga. Una vez identificado éste, se procede a su oclusión externa mediante ligadura de $2 / 0$, por debajo de los orificios ureterales. Cuando esta ligadura ha sido realizada se retira la sonda vesical. Se mantiene la obstrucción durante cuatro semanas.

El grupo Control de conejos es el grupo de animales sanos, no operados, es decir sin obstrucción vesical creada quirúrgicamente.

A las cuatro semanas ambos grupos de conejos son sacrificados y sometidos a cistectomía. La vejiga tras la cistectomía es sometida a dos tipos de estudios (Figs. 1 y 2):

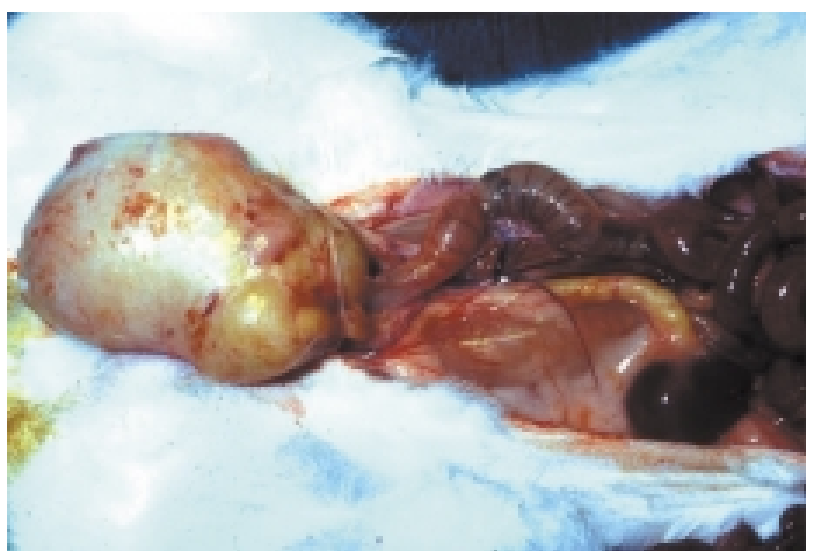

FIGURA 1. Vejiga del conejo sacrificado para realizar la cistectomia.

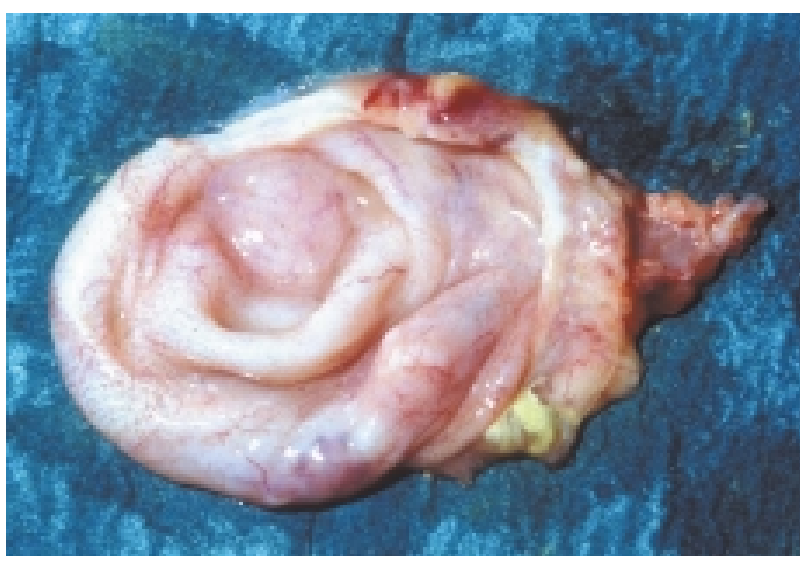

FIGURA 2. Vejiga del conejo tras cistectomia, abierta, preparada para estudios histopatológicos y fisiofarmacológicos.

1. Anatomopatológico, en la búsqueda de cambios histológicos acontecidas en el detrusor.

2. Estudios fisiofarmacológicos "in vitro" en "baños de órganos" (solución de Krebs) a T ” $37^{\circ} \mathrm{C}$, mediante fármacos agonistas y antagonistas de $\alpha_{1}$-adrenoceptores (Noradrenalina y Epinefrina) y estudios de contractilidad muscular.

Los agonistas empleados son Fenilefrina y Noradrenalina, y los antagonistas: WB4101, AH1 1101, BMY7378 (antagonistas respectivos de receptores $\alpha 1 \mathrm{a}, \mathrm{b}, \mathrm{d})$.

La segunda fase del trabajo de Investigación, sobre el paciente varón con HBP, será motivo de una nueva presentación a la obtención de resultados.

\section{RESULTADOS}

Analizado inicialmente el detrusor desde el punto de vista anatomo-patológico se demuestra que existe mayor grosor de la pared vesical en los casos de obstrucción vesical (hipertrofia del detrusor) en comparación con el detrusor de los conejos control (no obstruidos).

De los estudios isométricos y fisiofarmacológicos se han obtenido los siguientes datos, que vienen resumidos en cuatro puntos y reflejados en las tablas y gráficas que se adjuntan. Los datos han sido evaluados estadísticamente siendo significativos aplicando la t de student.

1. En conejos obstruidos (Fig. 3) la población de receptores $\alpha 1$ está incrementada, mientras 


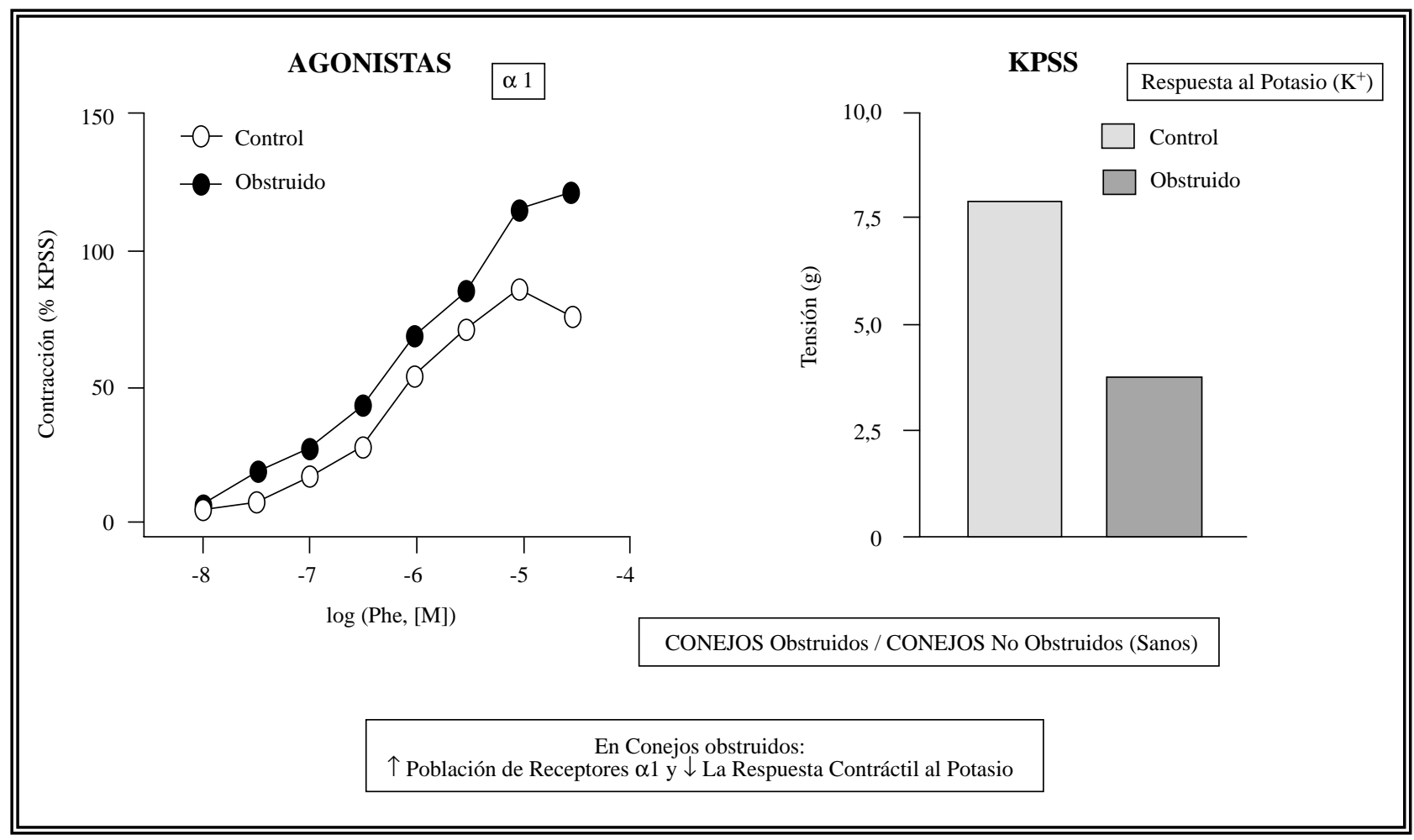

\section{FIGURA 3}

que la respuesta contráctil al potasio (KPSS) está disminuida cuando se comparan las respuestas con las obtenidas en el grupo de animales sanos (control).

\begin{tabular}{|l|l|l|}
\hline & $\begin{array}{l}\text { Phenilefrina } \\
\text { (Emax) }\end{array}$ & KPSS \\
\hline Grupo control & $88,3 \pm 5,6 \%$ & $7 \pm 0,9 \%$ \\
\hline $\begin{array}{l}\text { Conejos } \\
\text { obstruidos }\end{array}$ & $108,7 \pm 10,4 \% *$ & $4,2 \% \pm 0,6 \% * *$ \\
\hline
\end{tabular}

${ }^{*} \mathrm{p}<0,05$ significativo.

${ }^{* *} \mathrm{p}<0,01$ muy significativo, aplicando t de student.

2. Los receptores $\alpha$ la son posiblemente, los subtipos de receptores activos tanto en condiciones normales como en la obstrucción.

En ambos grupos de animales, el bloqueo (WB4101) de estos receptores es muy similar, por lo tanto, no parece que exista un cambio en la población de receptores $\alpha$ la tras la obstrucción.

3. En relación a los receptorres $\alpha 1 \mathrm{~b}$ parecen no actuar en la contracción del detrusor.
En ambos grupos de animales el bloqueo con el antagonista específico (AH11101) no provoca ningún cambio en la curva dosis-respuesta al agonista fenilefrina, por lo tanto, este subtipo de receptor parece no actuar en la contracción de la vejiga del conejo.

4. En relación a los receptores $\alpha 1 \mathrm{~d}$ se observan cambios muy importantes en la población de conejos sometidos a obstrucción quirúrgica.

En el grupo control (animales sanos) no se observan apenas cambios tras su bloqueo. Sin embargo si que se observan, en el grupo de conejos obstruidos, un aumento de la población de estos receptores (BMY7378) (Fig. 4).

\section{DISCUSIÓN}

La Investigación Farmacológica permitió ya en los años 60 establecer un mapa de receptores autonómicos en la vejiga y el tracto urinario inferior. Actualmente se conoce que los receptores colinérgicos y los $\beta$-adrenérgicos dominan en la región del cuerpo vesical y los receptores $\alpha$-adrenérgicos están fundamentalmente presentes en la región de la base de la vejiga y la uretra $^{2}$. 


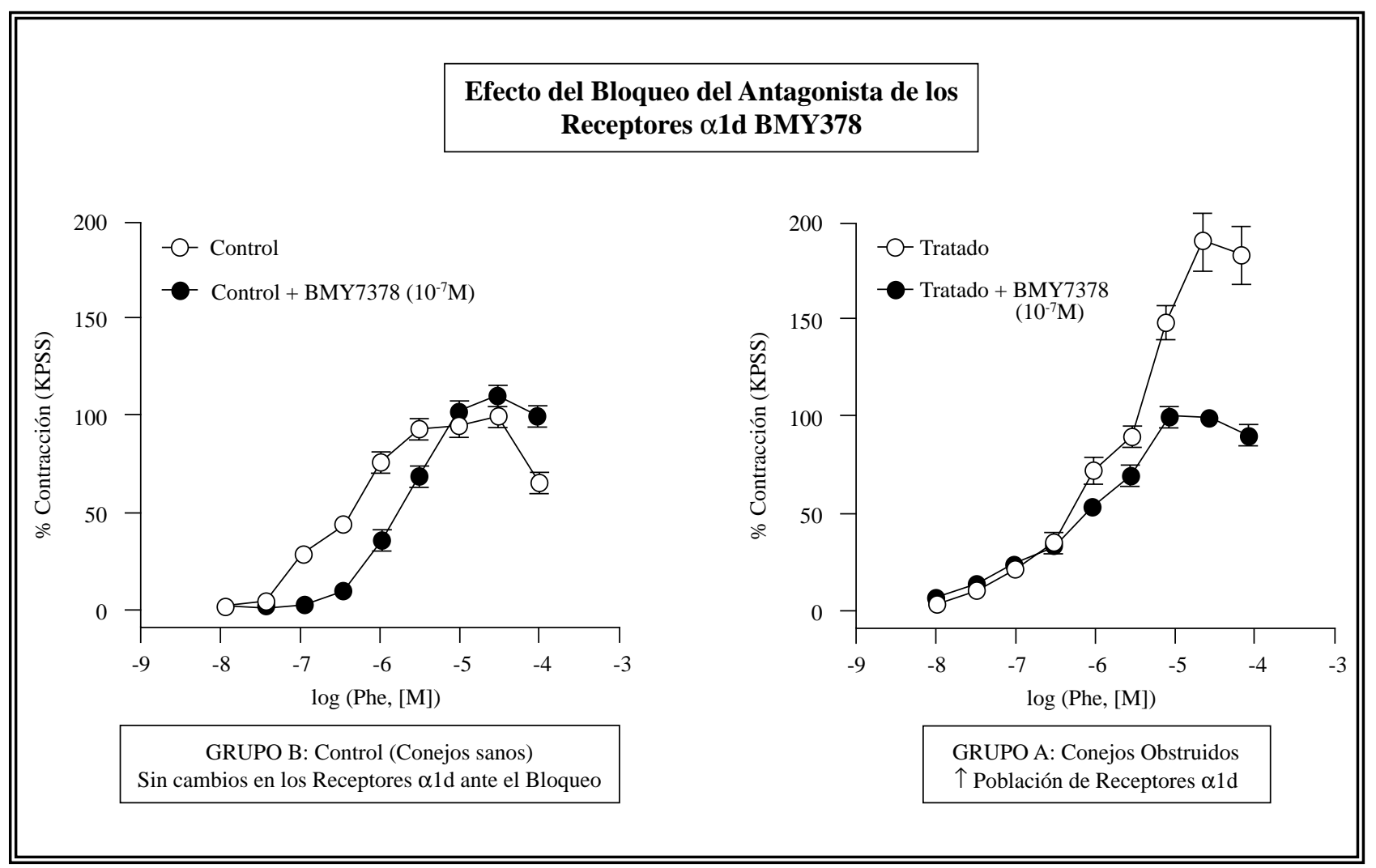

FIGURA 4

Hasta estos años la micción estaba basada en conceptos mecánicos y estáticos. Desde estos hallazgos la micción se configura como un proceso dinámico con un fundamento neurohormonal, concepto que se enfrenta a las teorías mecanicistas tan en boga en tiempos anteriores. La existencia de receptores adrenérgicos en el área cervicouretral, y la posibilidad de manipular farmacológicamente este área, ha sido ampliamente utilizado, durante estos años, en el manejo y tratamiento de las disfunciones miccionales neurógenas $^{1-4}$.

La dinámica miccional se establece en dos fases, la fase de llenado y la fase de vaciado, controladas ambas por impulsos simpáticos y mecanismos colinérgicos.

En lo que se refiere a la actividad simpática en la vejiga durante la micción, y en la fase de llenado, a través de la vía $\beta$-receptores, se produce una inhibición de la contracción del detrusor, es decir, una relajación del músculo que permite aumentar la capacidad vesical sin aumentar la presión. Simultáneamente y través de la vía $\alpha$ - receptores se produce un aumento de la resistencia del trígono y de la uretra, por contracción del músculo liso, lo que dificulta la salida de orina de la vejiga. Por ambas vías y por mecanismos complementarios se consigue el control de la continencia ${ }^{9}$.

El protagonismo del simpático, conocido inicialmente, como responsable exclusivo de la eyaculación, se constata con estos avances, de su extraordinaria importancia en la dinámica miccional y puede asegurarse que una vez establecido el concepto neurohormonal de la micción y de la continencia, la manipulación farmacológica del simpático, ha sido mucho más rentable farmacológicamente hablando, en las disfunciones vesicales neurógenas que lo correpondiente al parasimpático ${ }^{10,11}$.

Es posterior en el tiempo, avanzada la década de los años setenta, y mediante técnicas isométricas, cuando se reconocieron receptores $\alpha$ adrenérgicos en la próstata: en el área cérvicoprostática, en el adenoma y la cápsula prostática, así como la posibilidad de manipulación far- 
macológica de esta zona, en pacientes con prostatismo ${ }^{12-14}$. Estos avances motivaron un notable éxito clínico y un extraordinario entusiasmo investigador tanto a nivel básico como de farmacología clínica.

Con el mismo razonamiento establecido en la micción, se ha podido establecer que la obstrucción prostática, no está motivada exclusivamente por la presencia física del adenoma en el canal uretral sino también por el aumento de tono de todos los elementos incluidos en este área, especialmente del músculo liso del adenoma. Y por ello como pasó con la micción, se establece un concepto dinámico del prostatismo, frente al mecanicista clásico y anterior. Estos hallazgos motivaron los primeros ensayos clínicos farmacológicos con fenoxibenzamina y otros $\alpha$-antagonistas no específicos ${ }^{1}$.

Se conoce que los receptores $\alpha$-adrenérgicos son de dos tipos: los $\alpha 1$ y $\alpha 2$. De los receptores $\alpha 1$, de estimulación simpática (adrenérgica), recientemente se han identificado tres subtipos: $\alpha 1 \mathrm{a}, \alpha 1 \mathrm{~b}$ y $\alpha 1 \mathrm{~d}$. Todos ellos se han clonado, se conoce su ubicación, y el gen que codifica a cada uno de ellos, pero no se conoce aún la existencia de funciones específicas asociadas a cada subtipo de receptor $\alpha$. El $\alpha$ la prevalece en el tejido prostático y cuello de la vejiga, el $\alpha \mathrm{lb}$ es un receptor vascular y el $\alpha 1 \mathrm{~d}$ se ha identificado en el detrusor, en la vejiga ${ }^{15-18}$.

Mediante estudios in vivo en animales de experimentación (perros) y en el hombre (paciente prostático) se conoce que, la obstrucción vesical crónica provoca hipertrofia del detrusor y un neto incremento de $\alpha$-adrenoceptores ${ }^{19-20}$. Es clínicamente conocido que mediante fármacos $\alpha 1$ bloqueantes (antagonistas), es decir, que bloquean la acción del simpático a nivel de los receptores $\alpha 1$ del músculo liso cérvico-uretral y del adenoma prostático, podemos mejorar de manera considerable la sintomatología del paciente que sufre una obstrucción prostática ${ }^{21}$. Esta respuesta observada puede ser debida a la acción relajante de la obstrucción dinámica de la próstata. La industria farmacéutica está vinculizada a desarrollar fármacos altamente selectivos correspondientes al subtipo $\alpha$ la con el objetivo de mejorar la eficacia disminuyendo los efectos adversos de los mismos.
Pero de manera paralela a esta evidencia clínica de mejoría sintomática, los $\alpha$-bloqueantes no mejoran de manera similar a todos los pacientes, ni mejoran de manera tan llamativa el flujo miccional. La densidad de $\alpha 1$-adrenoceptores es similar en la cápsula de pacientes con y sin HBP, pero la respuesta farmacológica a $\alpha$-antagonistas puede que sea diferente. Estas variaciones clínicas entre pacientes podrían ser debidas a diferencias de sensibilidad de adrenoceptores (hipersensibilidad) más que ha diferencias cuantitativas ${ }^{4}$.

En cuanto a la escasa mejoría del flujo miccional, posiblemente parte de la acción farmacológica de los $\alpha$-antagonistas, se deba a la acción sobre los adrenoceptores del detrusor. La mejoría del síndrome irritativo, tan peculiar de la inestabilidad vesical consecuente con la obstrucción prostática $^{22-24}$, podría ser explicada con estos conocimientos tan recientes.

Si bien el papel funcional de estos receptores no ha sido claramente establecido hasta la actualidad, si que parece que pueden sufrir cambios en cuanto a su número y función en diferentes momentos de la actividad funcional del tracto urinario como lo demuestra la obstrucción vesical al flujo, la hiperactividad vesical o en la vejiga neurógena ${ }^{25}$. Se piensa que los receptores $\alpha$ del detrusor, sobre todo en HBP (obstrucción prostática) juegan un especial protagonismo ${ }^{26}$. Hasta el momento se conoce que en el detrusor existen dos subtipos de receptores $\alpha 1: \alpha 1 \mathrm{~d}$ y $\alpha$ la, siendo el $\alpha 1 d$ el más común. Si esta apreciación se confirmase, para corregir el síndrome irritativo de la uropatía obstructiva, tendría más sentido utilizar $\alpha$-antagonistas selectivos $\alpha 1 \mathrm{~d}$ que $\alpha$ la, hipótesis que aún no ha sido confirmada.

Esta evidencia clínica que objetivamos diariamente en los pacientes prostáticos tratados farmacológicamente con $\alpha$-antagonistas, nos ha llevado a diseñar el proyecto de investigación básica que se presenta y cuya hipótesis de trabajo está dirigida a investigar la cuantía y signo de la población de receptores $\alpha$-adrenérgicos a nivel de detrusor en pacientes que sufren patología obstructiva como consecuencia de HBP.

Los resultados obtenidos de las técnicas isométricas y estudios fisiofarmacológicos indican que efectivamente como consecuencia de la obs- 
trucción prostática se originan una serie de cambios en la población de receptores $\alpha$ del detrusor. La población de receptores $\alpha$ en su conjunto, aumenta en número en el grupo de conejos obstruidos. Los receptores $\alpha$ la parecen ser los subtipos de receptores activos tanto en condiciones normales como en la obstrucción y sin embargo no aumentan en número en el grupo de conejos obstruidos. Los receptores $\alpha \mathrm{lb}$ no parecen actuar en este proceso. Y son los receptores $\alpha 1 \mathrm{~d}$ los que realmente aumentan en el detrusor vesical, de manera significativa, durante la obstrucción en comparación con el grupo control no sometido a obstrucción quirúrgica. Paralelamente como consecuencia de la obstrucción, los cambios anatomo-patológicos que sufre el detrusor (hipertrofia) se corresponden con una respuesta contráctil al potasio disminuida.

Estos datos coinciden con los resultados de otros grupos de trabajo que han investigado la presencia y la función de los diferentes receptores $\alpha 1$-adrenérgicos (subtipos) en otros sistemas orgánicos (circulatorio, sistema nervioso central,...) y zonas extraprostáticas (vejiga,...), mediante técnicas de reacción en cadena de la polimerasa (PCR) ${ }^{27,28}$. El detrusor expresa predominantemente los subtipos $\alpha 1 \mathrm{~d}$, en menor medida $\alpha 1$ a, y no expresa $\alpha 1 b$.

En el estudio de Hampel (2000) ${ }^{29}$, realizado con ratas hembras, después de la obstrucción al flujo, había una tendencia a un aumento de la expresión de los $\alpha 1$ totales que no fue estadísticamente significativa. Sin embargo, si es muy importante que, hubo un cambio en la expresión del subtipo. La expresión de los $\alpha$ la se redujo desde un $70 \%$ a un $5 \%$, mientras que existió un aumento de la expresión de los ald desde un $16 \%$ a un $91 \%^{29}$.

La segunda parte de esta etapa inicial sobre modelo animal (conejos), primera parte del estudio, está desarrollándose. Todavía sobre modelo quirúrgico animal, se están realizando estudios "in vivo" mediante polígrafo. La segunda parte del proyecto se realizará en pacientes varones con patología prostática obstructiva con el mismo objetivo de trabajo y comparándolo con varones sanos (control).

Para mayor complejidad, en el capítulo de análisis de resultados, si por una parte es cono- cido que la función exacta de los diferentes subtipos de receptores $\alpha$ no está totalmente identificada, sí que parece estar demostrado, que su acción puede variar según las especies animales estudiadas $^{27}$.

Si las hipótesis de trabajo establecidas en la investigación que se presenta, sobre modelo quirúrgico animal, se confirmasen en la segunda parte del estudio, para tratar la sintomatología de la HBP, la Investigación Farmacológica a través de su Industria, no sólo tendría que pensar en el desarrollo de fármacos $\alpha 1$-antagonistas dirigidos hacia los receptores $\alpha$ la (próstata), sino también en otras moléculas que actuasen sobre los receptores $\alpha 1 \mathrm{~d}$ (vejiga) que como acabamos de demostrar son realmente los que están aumentados en situaciones de obstrucción al flujo vesical (HBP), localizados a nivel del detrusor, y responsables de la sintomatología irritativa del prostatismo por hiperactividad consecuente de la vejiga ${ }^{30}$.

\section{CONCLUSIONES}

1. La sintomatología del prostatismo está co-protagonizada por el comportamiento de la vejiga. El síndrome irritativo (urgencia miccional, polaquiuria, nocturia,...) es la traducción clínica de la inestabilidad vesical que origina la obstrucción al flujo miccional (obstrucción prostática). Estos cambios funcionales y patológicos que sufre la vejiga en condiciones de obstrucción, están modulados por los receptores $\alpha$-adrenérgicos, aunque la función de éstos y de los subtipos de receptores $\alpha 1$ ( $\alpha$ la,b,d), no ha sido claramente establecida en el detrusor normal.

2. Parece evidente, tras la observación y el análisis de resultados de esta investigación, en modelo quirúrgico de animal de experimentación, que existe un cambio poblacional de receptores $\alpha$ en el detrusor del conejo, sometido a obstrucción, a favor de los receptores $\alpha 1$ d.

3. En el tratamiento medicamentoso de la HBP, no sólo se deberá corregir la obstrucción o disminuir el tamaño de la próstata, si no que además se deberá atender el comportamiento de la vejiga, el síndrome irritativo provocado por HBP, la inestabilidad vesical consecuente de la obstrucción prostática. 
4. Para estos fines, y según la experiencia diseñada y sus resultados, a expensas de obtener resultados de la experiencia animal "in vivo" y humana, que se está desarrollando en la actualidad, la Investigación Farmacologica a través de la Industria deberá trabajar con un doble objetivo: fármacos que actúen sobre los receptores $\alpha$ la (adenoma de próstata, área cérvico-uretral, cápsula prostática,...), y fármacos que actúen sobre los receptores $\alpha 1 \mathrm{~d}$ que se encontrarian principalmente en el detrusor y muy aumentados en número, en condiciones de obstrucción al flujo miccional (prostatismo y HBP).

\section{REFERENCIAS}

1. LEVIN, RM, SHOFER FS, WEIN AJ.: Cholinergic, adrenergic and purinergic response of sequential strips of rabbit urinary bladder. J Pharmacol Exp Ther 1980; 212: 536.

2. LEVIN RM, WEIN AJ.: Distribution and funtion of adrenergic receptors in the urinary bladder of the rabbit. Mol Pharmacol 1979; 16: 441.

3. MALLOY BJ, PRICE DT, PRICE RR et al.: $\alpha 1$-adrenergic receptor subtypes in human detrusor. J Urol 1998 september; 160: 937-943.

4. HAMPEL C, DOLBER CP, SMITH MP et al.: Modulation of bladder $\alpha 1$-adrenergic receptor subtype expression by bladder outlet obstruction. J Urol 2002 march; 167: 1513-1521.

5. SEAMAN EK, JACOBS BZ, BLAIVAS JG et al.: Persistence or recurrence of symptoms after transurethral resection of the prostate: a urodynamic assessment. J Urol 1994; 152: 935 .

6. FORRAY C, BARD JA, WETZEL JM et al.: The $\alpha-1$ adrenergic receptor that mediates smooth muscle contraction in human prostate has the pharmacological properties of the cloned $\alpha$ lc subtypes. Mol Pharmacol 1994; 45: 703.

7. CALVERT RC, THOMPSON CS, KHAN MA et al.: Alterations in cholinergic and purinergic signaling in a model of the obstructer bladder. J Urol 2001 october; 166: 1530-1533.

8. WOODS M, CARSONN N, WESLEY N et al.: Efficacy of the beta3-adrenergic receptor agonist Cl-316243 on experimental bladder hyperreflexia and detrusor instability in the rat. J Urol 1997 november; 158: 1973-1977.

9. VELA NAVARRETE, R, RAZ, S.: Control farmacológico de los transtornos urodinámicos. Libro. Ed Científico Médica. Barcelona 1979.

10. CAINE M, RAZ S, ZIEGLER M et al.: Adrenergic and colinergic receptors in the human prostate prostatic capsule and bladder neck. Br J Urol 1975; 2 (27): 193-202.

11. CAINE M.: The importance of adrenergic receptors in disorders of micturition. Eur Urol 1977; 3 (1): 1-6.

12. RAZ S, ZIEGLER M, CAINE M.: Pharmacologic receptors in the prostate. Br J Urol 1975, 27: 193-202.

13. VELA NAVARRETE R.: Farmacología Neurohumoral del prostatismo. II Seminario de Farmacologia Urológica: "Farmacología de las disfunciones miccionales". "Fundación Jiménez Díaz". Cátedra y Servicio de Urología (Prof. R. Vela Navarrete). Universidad Autónoma. Madrid. Ed. Silabus. Enero 1995.

14. LEPOR H, SAPHIRO E.: Alpha 1-adrenergic receptor in lower genitourinary tissues: insight into development and funtion. J Urol 1987; 138: 979-983.
15. KOBAYASHI S, TANG R, SAPHIRO E, LEPOR H.: Characterization of human alpha 1 adrenoreceptor binding sites using radioligand receptor binding an slidemounted tissues sections. J Urol 1984; 150: 2002-2006.

16. XIAO-PING YANG \& SHIGETOSHI CHIBA.: Existence of different $\alpha 1$-adrenoceptor subtypes in junctional and extrajunctional neurovascular regions in canine splenic arteries. Britisth Journal of Pharmacology 2001; 132: 1852-1858.

17. SHOICHI UEDA, NOUBUHIRO SATAKE, SHOJI SHIBATA.: $\alpha 1$-and $\alpha 2$-adrenoceptores in the smooth muscle of isolated rabbit urinary bladder and uretra. European Journal of Pharmacology 1984; 103: 249-254.

18. ELTZE M, KÖNIG H, ULLRICH B, GREBE T.: Failure of AH111OA to functionally discriminate between 1-adrenoceptor subtypes A,B and D or between $\alpha 1$-and $\alpha 2$-adrenoceptors. European Journal of Pharmacology 2001; 415 : 265-276.

19. SCHRÖDER A, CHICHERTER P, KOGAN BA et al.: Effect of chronic bladder outlet obstruction on blood flow of the rabbit bladder. $J$ Urol 2001 february; 165: 640-646.

20. TONG YAT-CHING, HUNG YING-CHO, SHINOZUKA K et al.: Evidence of adenosine 5-triphosphate release from nerve and $\mathrm{p} 2 \mathrm{x}$-purinoceptor mediated contraction during electrical stimulation of rat urinary bladder smooth muscle. J Urol 1977 nov.; 158: 1973-1977.

21. VELA NAVARRETE R.: BPH alpha blocker therapy. Journal d'Urologie 1995; 101 (1): 26-28.

22. SÁNCHEZ CHAPADO M, ESTEBAN FUERTES M.: Respuesta vesical a la obstrucción: prostatismo. Med Clin (Barc) 2002; 118 (Supl 2): 10-12.

23. ESTEBAN FUERTE M, SALINAS CASADO J, MARTÍN PÉREZ M.: La inestabilidad vesical en las alteraciones de la fase de llenado vesical del varón. Urodinamia ilustrada. Madrid. Datagrafic, S.L, 1998; 17: 84-198.

24. PERLBERG S, CAINE M.: Adrenergic response of bladder muscle in prostatic obstruction. Its relation to detrusor inestability. Urology 1982 nov; 20 (5): 524-527.

25. ANDERSSON KE.: $\alpha 1-$ adrenoceptors and bladder function. European Urology 1999; 36 (suppl, 1): 96-102.

26. MARTÍN CM.: Protecting bladder function and reducing disease progression. Abstract. Managing LUTS/BPH: the total approach. XVIIIth Congress of the eau. Madrid, march, 2003.

27. RUDNER XL, BERKOWTZ DE, BOOTH JV et al.: Subtype specific regulation of human vascular $\alpha 1$-adrenergic receptors by vessel bed and age. Circulation. Journal of American Heart Association 1999 december 7; 100 (23): 1999.

28. SCHWINN DA.: Posible papel de los diferentes antagonistas de los receptores $\alpha 1$-adrenérgicos en los sintomas del tracto urinario inferior. Drugs of Today 2001; 37 (Supl. 14): 13-16.

29. HAMPEL C, DOLBER PC, SAVIC SL et al.: Changes in $\alpha 1-$ adrenergic receptor (AR) subtype gene expression during bladder outlet obstruction of rats. J Urol 2000; 163: 228 (abstract 1015).

30. VELA NAVARRETE R, CASTILLÓN VELA I, CALAHORRA FERNÁNDEZ FJ, GARCÍA CARDOSO J.: El tratamiento de la hiperplasia benigna de próstata en el futuro próximo: cambios previsibles. Med Clin (Barc) 2002; 118 (Supl 2): 35-40.

Dra. C. González Enguita

C/ Costa Brava, 26 - Blq. 2 - 3o A

28034 Madrid

(Trabajo recibido el 7 mayo de 2003) 